Chest 2000; 117: 551-5.

7 Gran T, Leipold RW, Horter J, Martin E, Motsch J. Colour Doppler imaging of the interspinous and epidural space. Eur J Anaesthesiol 2001; 18: 706-12.

\section{Analgesic effect of intra-articular tra- madol compared to morphine after arthroscopic knee surgery}

To the Editor:

Intra-articularly administered morphine has been studied extensively and shown to have a definite but mild analgesic effect. ${ }^{1}$ Although parenteral tramadol, an analgesic drug with some $\alpha_{2}$-adrenergic and opioid receptor agonist activity, produces analgesia equivalent to parenteral morphine in moderate postoperative pain, the efficacy of intra-articular (IA) tramadol remains unclear. ${ }^{2}$ We compared the analgesic effect of IA morphine with IA tramadol. Seventy-five patients, having elective arthroscopic surgery of the knee were randomized to receive either IA tramadol $50 \mathrm{mg}$, IA morphine $5 \mathrm{mg}$ or IA normal saline $20 \mathrm{~mL}$. The tourniquet was released ten minutes after analgesic administration. Exclusion criteria were preoperative narcotic use, contraindication to narcotics, seizure disorder, anterior cruciate ligament reconstruction, and traumatic injury to the knee.

We did not observe any differences among the three groups with respect to age, gender, weight, duration of surgery, surgical procedures, laser use during surgery, duration and severity of preoperative knee pain, time to discharge or side effects. There was also no difference with respect to pain scores or the use of rescue analgesics postoperatively, except for the first pain score when the patients arrived to the postanesthesia care unit and the time to first rescue analgesic (Table).

The dose and volume of morphine used in this study was based on our previous experience. ${ }^{3} \mathrm{We}$ believe duration and severity of the preoperative symptoms and pain can change the effectiveness of the postoperative analgesic. There was a good correlation between the duration of preoperative pain symptoms and time to first analgesic requirement after the operation $\left(\mathrm{r}^{2}=0.731, P<0.0001\right)$. Experimental and clinical evidence shows that analgesic effects of peripheral opioids and the density of opioid receptors on peripheral sensory nerves increase with the duration of painful inflammatory processes. ${ }^{4}$ After examining different time intervals, in the subgroup of patients with more than six months of preoperative pain (14 patients in the morphine and the control groups, ten patients in the tramadol group), the analgesic effects of IA narcotics were more prominent (Table). These patients benefited from the IA administration of tramadol or morphine, having had a chance to increase the expression of peripheral receptors.

We conclude that morphine and tramadol, at the doses used, provide similar analgesia when given intraarticularly. The mechanisms of action of IA opioids are yet to be elucidated. ${ }^{1}$ Further studies are needed to compare the adverse effects between IA morphine and tramadol. We believe IA tramadol can be an alterna-

TABLE Patient data

\begin{tabular}{llll}
\hline & Control $(n=25)$ & Morphine $(n=25)$ & Tramadol $(n=25)$ \\
\hline Age $(\mathrm{yr})$ & $41 \pm 17$ & $34 \pm 15$ & $34 \pm 17$ \\
VRS at rest $(0 \mathrm{~min})$ & $7(4-10)$ & $1(0-9)^{*}$ & $0(0-9)^{*}$ \\
VRS at rest $(15 \mathrm{~min})$ & $6(0-10)$ & $5(0-10)$ & $5(0-9)$ \\
VRS at rest $(30 \mathrm{~min})$ & $6(1-10)$ & $5(1-9)$ & $6(1-9)$ \\
VRS at rest $(45 \mathrm{~min})$ & $6(0-10)$ & $4(1-9)$ & $6(1-9)$ \\
VRS at rest (1st hr) & $5(0-9)$ & $4(0-9)$ & $5(0-8)$ \\
VRS at rest (2nd hr) & $3(0-8)$ & $3(0-9)$ & $4(0-8)$ \\
VRS at rest (6th hr) & $4(0-5)$ & $3(0-8)$ & $2(0-8)$ \\
VRS at rest (24th hr) & $2(0-7)$ & $1(0-9)$ & $1(0-7)$ \\
Time to first analgesic (min) & $25(15-55)$ & $34(15-158)^{*}$ & $33(17-728)^{*}$ \\
Time to first analgesic (preoperative pain $\geq 6$ months) & $25(15-45)$ & $35(15-160)^{*}$ & $60(26-746)^{*}$ \\
No analgesics needed & $3(12 \%)$ & $5(20 \%)$ & $5(20 \%)$ \\
Opioids needed in PACU & $18(72 \%)$ & $13(52 \%)$ & $12(48 \%)$ \\
Opioids needed in PACU (preoperative pain $\geq 6$ months) & $12(86 \%)$ & $8(57 \%)^{*}$ & $3(30 \%)^{*}$ \\
Number of opioid boluses & $1(0-5)$ & $1(0-3)$ & $1(0-3)$ \\
Dipyrone needed in $24 \mathrm{hr}$ & $4(16 \%)$ & $7(28 \%)$ & $8(32 \%)$ \\
Tablets of dipyrone in $24 \mathrm{hr}$ & $2(0-5)$ & $1(0-3)$ & $1(0-7)$
\end{tabular}

Pain was assessed by verbal rating score between 0-10 (VRS). Results are given as number of patients (\%), mean \pm SD or median $(95 \%$ $\mathrm{CI}) . \mathrm{PACU}=$ postanesthesia care unit; ${ }^{*} P<0.008$ compared to control, $P>0.05$ between morphine and tramadol. 
tive to IA morphine for postoperative analgesia after arthroscopic surgery, especially in patients with more than six months of preoperative pain.

Seda B. Akinci MD

Fatma Saricaoglu MD

Ahmet Atay MD

Mahmut N. Doral MD

Meral Kanbak MD

Ankara, Turkey

\section{References}

1 Gupta A, Bodin L, Holmstrom B, Berggren L. A systematic review of the peripheral analgesic effects of intraarticular morphine. Anesth Analg 2001; 93: 761-70.

2 Power I, Barratt S. Analgesic agents for the postoperative period. Nonopioids. Surg Clin North Am 1999; 79: 275-95.

3 Kanbak M, Akpolat N, Ocal T, Doral MN, Ercan M, Erdem $K$. Intraarticular morphine administration provides pain relief after knee arthroscopy. Eur J Anaesthesiol 1997; 14: 153-6.

4 Likar R, Schafer M, Paulak F, et al. Intraarticular morphine analgesia in chronic pain patients with osteoarthritis. Anesth Analg 1997; 84: 1313-7.

\section{Low-dose epidural anesthesia for cervi- cal cerclage}

To the Editor:

McDonald cervical cerclage is an obstetric outpatient procedure in the lithotomy position frequently performed under spinal anesthesia (SA). Recently, lowdose SA techniques facilitating intraoperative hemodynamic stability and fast recovery have been described for this and other outpatient procedures. ${ }^{1-3}$ However, undesirable outcomes of SA include transient neurologic symptoms (TNS) from local anesthetic toxicity particularly for procedures in the lithotomy position $^{4,5}$ and nausea and pruritus from adjuvant intrathecal opioids. ${ }^{1}$ Low-dose, or differential epidural anesthesia has not been described for McDonald cerclage as an alternative technique. A healthy 41 -yr-old female, gravida 2 para 0 , presented for the above procedure at $135 / 7$ weeks gestation. Based on her prior anesthetic experience she requested regional anesthesia that would provide a gradual onset and minimal motor blockade. After initiation of electrocardiogram, noninvasive blood pressure and oxygen saturation monitoring in the operating room an $18-\mathrm{G}$ epidural catheter was placed in the L 3-4 interspace. A negative test dose of $3 \mathrm{~mL}$ of $0.125 \%$ bupivacaine with epinephrine $1: 200,000$ was followed by incremental epidural administration of a total of $20 \mathrm{~mL}$ of bupivacaine $0.125 \%$ with $50 \mu \mathrm{g}$ of fentanyl added to the first $10 \mathrm{~mL}$ of solution. A sensory level to T4 by cold sensation was apparent at 15 min. During placement of the epidural catheter and the subsequent 26 min surgery the patient received $50 \mu \mathrm{g}$ of fentanyl intravenously in divided doses. Blood pressure, heart rate and oxygen saturation remained within normal limits and free movement of the legs (Bromage 0) was preserved during the procedure. The patient and her anesthesiologist maintained a conversation throughout. The surgeon rated operating conditions as satisfactory. The patient easily moved herself to the stretcher postoperatively and was highly satisfied with her anesthetic. No nausea or pruritus occurred. She was discharged uneventfully after a fourhour bedrest dictated by the obstetric protocol. This case shows that low-dose epidural anesthesia can be an alternative to SA for selected patients presenting for McDonald cerclage. Advantages include the absence of the risk for TNS, and possibly a reduced incidence of perioperative nausea and pruritus. The technique could be improved by using less volume of local anesthetic solution to achieve the T8 level of sensory blockade that suffices for this surgery. Placement of an epidural catheter perceived as cumbersome for outpatients may not be needed when employing a single-shot epidural technique instead. Comparative outcome studies are desirable.

Roman Schumann MD

Muhammad B. Rafique MD

Boston, Massachusetts

\section{References}

1 Richardson M, Wissler R, Porth J. Cervical cerclage: spinal lidocaine vs bupivacaine/fentanyl.

Anesthesiology 1998; 88 (SOAP Suppl.): A 32 (abstract).

2 Zahn J, Abramovitz SE. Subarachnoid fentanyl with low dose bupivacaine vs. lidocaine for cervical cerclage. Anesthesiology 1999; 90 (SOAP Suppl.): A 24 (abstract).

3 Ben-David B, Solomon E, Levin H, Admoni H, Goldik $Z$. Intrathecal fentanyl with small-dose dilute bupivacaine: better anesthesia without prolonging recovery. Anesth Analg 1997; 85: 560-5.

4 Freedman JM, Li DK, Drasner K, Jaskela MC, Larsen $B$, Wi S. Transient neurologic symptoms after spinal anesthesia: an epidemiologic study of 1,863 patients. Anesthesiology 1998; 89: 633-41. 